\title{
Joint Medium Access Control, Routing and Energy Distribution in Multi-Hop Wireless Networks
}

\author{
Khoa T. Phan, Student Member, IEEE, Hai Jiang, Member, IEEE, Chintha Tellambura, Senior Member, IEEE, \\ Sergiy A. Vorobyov, Senior Member, IEEE, and Rongfei Fan
}

\begin{abstract}
It is a challenging task for multi-hop wireless networks to support multimedia applications with quality-ofservice $(\mathrm{QoS})$ requirements. This letter presents a joint crosslayer optimization approach, i.e., joint medium access control, routing, and energy distribution. User satisfaction represented by user utility is maximized within the required network lifetime, given the constraints on the total available energy in the network and the minimum user rates. Although the resulting optimization problem is nonlinear and nonconvex, we prove that it is approximately equivalent to a two-step convex problem. Furthermore, we prove that the problem of maximizing network utility within achievable network lifetime is quasiconvex.
\end{abstract}

Index Terms-Cross-layer design, medium access control, routing, energy distribution, convexity.

\section{INTRODUCTION}

$\mathbf{I}$ $\mathrm{T}$ is a challenging task for multi-hop wireless networks (such as mobile ad hoc networks, wireless mesh networks, and wireless sensor networks) to support multimedia applications with quality-of-service (QoS) requirements. In these networks, each terminal may act as both a traffic source node and a relay node. Thus, it is necessary to design the medium access control (MAC) and routing protocols together, where MAC determines when a node transmits with what power and rate levels, and routing searches an effective path for an end-to-end flow [1], [2], [3]. Cross-layer design of MAC and routing has been well investigated in the literature [4]. Recently research has also shown the importance of energy distribution in multi-hop wireless networks [5]. It is well accepted that equal energy assignment among all nodes may not be efficient. For example, in a mobile ad hoc network with a wireless gateway, nodes located closer to the gateway might need more energy since they very likely have more traffic load (due to relay service) than others. Energy distribution strategies for wireless sensor networks are investigated in [5],

Manuscript received November 8, 2007; revised March 30, 2008 and July 4, 2008; accepted July 17, 2008. The associate editor coordinating the review of this letter and approving it for publication was G. Xue. This work was supported in part by the Natural Science and Engineering Research Council (NSERC) of Canada and Alberta Ingenuity, Alberta, Canada.

K. T. Phan was with the Department of Electrical and Computer Engineering, University of Alberta, Canada, and will be with the Department of Electrical Engineering, California Institute of Technology, Pasadena, CA 91125, USA (e-mail: kphan@caltech.edu).

H. Jiang, C. Tellambura, S. A. Vorobyov, and R. Fan are with the Department of Electrical and Computer Engineering, University of Alberta, Edmonton, Alberta, Canada T6G 2V4 (e-mail: \{hai.jiang, chintha, vorobyov\}@ece.ualberta.ca, rongfei@ualberta.ca).

S. A. Vorobyov is also a Honorary Reader with Joint Research Institute, Heriot-Watt and Edinburgh Universities, Edinburgh, U.K.

Digital Object Identifier 10.1109/T-WC.2008.071252 which largely depend on the locations of the nodes in the network.

This letter presents the joint design of MAC, routing and energy distribution in a multi-hop wireless network, where the QoS of each node must be guaranteed during the minimum required network lifetime, and the network utility within this lifetime is to be maximized. The wireless service provisioning is formulated as a nonconvex network utility maximization (NUM) problem. It is proved that the problem is approximately equivalent to a two-step convex problem. It is also proved that the NUM problem that maximizes the network utility within achievable network lifetime is a quasiconvex problem. The rest of this letter is organized as follows. Network model is given in Section II. The joint MAC, routing, and energy distribution design is presented in Section III. Numerical results are given in Section IV, followed by further discussions in Section $\mathrm{V}$ and concluding remarks in Section VI.

\section{NeTwORK MODEL}

A network with a node set $\mathcal{S}$ is considered. We assume that all the nodes have the same traffic destination (which is not included in $\mathcal{S} .{ }^{1}$ Each node in $\mathcal{S}$ acts as a traffic source node with relay function. Note that our simplified formulation can be easily extended to the case when any node has the function of both a traffic source and a traffic destination, since that node can be modeled as two nodes: one traffic source and one traffic destination at the same location.

We assume that each node has infinitely backlogged traffic ready to send. The rate of traffic source generated by a node $s \in \mathcal{S}$ and injected into the network is denoted $r_{s}$, which should be no less than a lower bound $r_{s}^{\mathrm{LB}}$. Note that this problem formulation can be also extended straightforwardly to the case when each node has a source rate upper bound.

The set of one-hop unidirectional links in the network is denoted as $\mathcal{L} .^{2}$ For each node $s$, let $\mathcal{O}(s)$ denote the set of outgoing links, and $\mathcal{I}(s)$ the set of incoming links. Then for node $s$, the difference of its total outgoing traffic and total incoming traffic should be exactly the traffic generated at $s$, that is

$$
\sum_{l \in \mathcal{O}(s)} R_{l}-\sum_{l \in \mathcal{I}(s)} R_{l}=r_{s}, \quad s \in \mathcal{S}
$$

\footnotetext{
${ }^{1}$ For example, in a wireless sensor network, the traffic sink collects information from all sensor nodes, and thus is the common traffic destination.

${ }^{2}$ Note that a bidirectional link in the network, if any, can be modeled as two unidirectional links.
} 
where $R_{l}$ is the transmission rate achieved over link $l$. Note that link $l$ may not be active (i.e., being transmitting) all the time. So $R_{l}$ is upper bounded by the capacity of link $l$ denoted $C_{l}$, which is the rate that link $l$ can achieve if the link is active all the time. The value of $C_{l}$ depends on the physical layer parameters such as spectrum bandwidth, modulation scheme, channel coding, etc.

For each link $l$, let $\epsilon_{l}$ and $\varepsilon_{l}$ denote the energy needed to transmit and receive a unit of traffic, respectively. Then the constraint on total energy consumption at node $s$ can be expressed as

$$
\left[\sum_{l \in \mathcal{O}(s)} \epsilon_{l} R_{l}+\sum_{l \in \mathcal{I}(s)} \varepsilon_{l} R_{l}\right] T_{s} \leq E_{s}, \quad s \in \mathcal{S}
$$

where $T_{s}$ means the lifetime of node $s$, and $E_{s}$ is the initial energy supply at node $s$. It is required that the QoS requirement of each node (i.e., $r_{s} \geq r_{s}^{\mathrm{LB}}$ ) should be guaranteed during the network lifetime, which is defined as the duration before any node dies. Therefore, the network lifetime can be expressed as

$$
T=\min _{s \in \mathcal{S}} T_{s}=\min _{s \in \mathcal{S}} \frac{E_{s}}{\sum_{l \in \mathcal{O}(s)} \epsilon_{l} R_{l}+\sum_{l \in \mathcal{I}(s)} \varepsilon_{l} R_{l}} .
$$

In this letter, low node mobility is assumed, such as in the case of a wireless mesh or sensor network. We consider the stage of node and network configuration. One target is to assign a certain amount of energy (e.g., a number of AAA batteries) to the wireless terminals based on the network topology and QoS requirements of the nodes. Different nodes should be allocated different amount of energy, since apparently equal energy assignment may not lead to optimality. The total available energy for the whole network is denoted $E_{\text {tot }}$, which can be in the form of available batteries with different capacities. With the assumption of continuous energy distribution, we have the following constraint in the network

$$
\sum_{s \in \mathcal{S}} E_{s} \leq E_{\text {tot }}
$$

\section{A. Link Contention Graph and Maximal Cliques}

Two popular and powerful tools to model contention relations in a multi-hop wireless network are link contention graph and maximal cliques [4], [6], [7], [8]. Each link in the network is represented by a vertex in the link contention graph. For two contending links in the network, an edge between the two associated vertices in the link contention graph is used to represent the contention relation. A clique is a subgraph in the link contention graph within which any two vertices have an edge (or equivalently, the two represented links in the network contend with each other). A maximal clique is defined as a clique not included in any other clique. It is possible that a link may belong to several maximal cliques. For a maximal clique, at most one link from it can be active at any time, because collision(s) will happen otherwise. Then we have

$$
\sum_{l \in \mathcal{M}_{k}} \frac{R_{l}}{C_{l}} \leq 1, \quad k=1, \ldots, K
$$

where $\frac{R_{l}}{C_{l}}$ is the fraction of time when link $l$ is active, $K$ is the total number of maximal cliques in the link contention graph, and $\mathcal{M}_{k}$ is the $k$ th maximal clique. Moreover, it is assumed here that the link contention graph is perfect. ${ }^{3}$ Therefore, the condition (4) is also a sufficient condition for a feasible schedule with link rates $R_{l}$ 's [7].

\section{Joint Design of MAC, Routing, AND Energy DISTRIBUTION}

\section{A. Problem Formulation}

This section presents a joint MAC, routing, and energy distribution optimization problem. The network lifetime $T$ is required to be at least $T_{\min }$.

The NUM framework [9] has been considered as a powerful tool for network rate allocation problems. In the framework, the utility denoted as $U_{\beta}\left(r_{s}\right)$ parameterized by $\beta \geq 0$ represents the user satisfaction level with respect to allocated resources (for example, traffic source rate $r_{s}$ ). We propose the following NUM problem

$$
\begin{array}{ll}
\left\{r_{s}\right\}, & \max _{\left\{R_{l}\right\},\left\{E_{s}\right\}, T} \quad \sum_{s \in \mathcal{S}} U_{\beta}\left(r_{s}\right) \\
\text { s.t. } & \sum_{l \in \mathcal{O}(s)} R_{l}-\sum_{l \in \mathcal{I}(s)} R_{l}=r_{s}, \quad s \in \mathcal{S} \\
& r_{s} \geq r_{s}^{\mathrm{LB}}, \quad s \in \mathcal{S} \\
& {\left[\sum_{l \in \mathcal{O}(s)} \epsilon_{l} R_{l}+\sum_{l \in \mathcal{I}(s)} \varepsilon_{l} R_{l}\right] T \leq E_{s}, s \in \mathcal{S}} \\
& T \geq T_{\min } \\
& \sum_{s \in \mathcal{S}} E_{s} \leq E_{\text {tot }} \\
& \sum_{l \in \mathcal{M}_{k}} \frac{R_{l}}{C_{l}} \leq 1, \quad k=1, \ldots, K .
\end{array}
$$

In this optimization problem, the objective is to maximize the total network utility for all nodes. Constraint $(5 b)$ means that a route exists for the traffic generated by any node. Constraint (5c) means that each node is guaranteed its minimum traffic source rate requirement. Constraint $(5 \mathrm{~d})$ specifies the total energy supply at each node. Constraint $(5 \mathrm{e})$ guarantees that the network lifetime should be at least $T_{\min }$. Note that the required minimum network lifetime $T_{\min }$ is determined based on the design goal of the network. ${ }^{4}$ Constraint (5f) specifies the total energy supply in the network. Constraint (5g) gives a sufficient and necessary condition for a feasible schedule with link rates $R_{l}$ 's, as indicated in the preceding section.

It has been shown that different utility definitions can result in different kinds of fairness [9], [10]. For example, in [10], a family of utility functions is given as

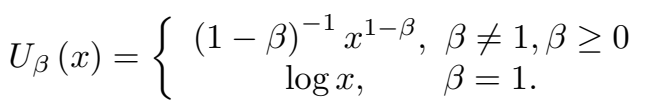

When $\beta=0$, the maximization of the network utility (i.e., the sum of the utility values of all the nodes in the network)

\footnotetext{
${ }^{3}$ Our formulation is also meaningful for a non-perfect link contention graph, since it can provide an upper bound of the network performance.

${ }^{4}$ In case when $T_{\min }$ is not designed appropriately or the total energy is not sufficient to achieve the $T_{\min }$, the problem (5a)-(5g) may be infeasible, such as in the case of Scheme 1 in Fig. 3 when $E_{\text {tot }}<40 \mathrm{~K}$ in Section IV.
} 
will lead to the maximization of system throughput. Moreover, proportional, harmonic mean, and max-min fairness can be achieved when $\beta=1, \beta=2$, and $\beta \rightarrow \infty$, respectively [7] [10]. Since such a utility definition is general and flexible enough, it is adopted here.

The aforementioned NUM problem $(5 \mathrm{a})-(5 \mathrm{~g})$ is both nonlinear and nonconvex. Thus it may look intractable. However, in the sequel, we develop an algorithm for solving it near optimally.

\section{B. Near Optimal Solution}

Let us consider the optimization problem (5a)-(5g) without the constraint on the minimum network lifetime, that is

$$
\begin{aligned}
& \max _{\left\{r_{s}\right\},\left\{R_{l}\right\},\left\{E_{s}\right\}, T} \sum_{s \in \mathcal{S}} U_{\beta}\left(r_{s}\right) \\
& \text { s.t. Constraints }(5 \mathrm{~b}),(5 \mathrm{c}),(5 \mathrm{~d}),(5 \mathrm{f}),(5 \mathrm{~g}) .
\end{aligned}
$$

THEOREM 1: If the network lifetime $T$ is fixed in (7a)(7b), then the optimal value of the optimization problem (7a) is non-increasing with respect to $T$.

Proof: Suppose that the network lifetime $T$ in (7a)-(7b) is fixed at $T_{f}$, and define $\tilde{E}_{s}=\frac{E_{s}}{T_{f}}$. After some mathematical manipulations, (7a)-(7b) can be reformulated as

$$
\begin{array}{ll} 
& \max _{\left\{r_{s}\right\},}\left\{R_{l}\right\},\left\{\tilde{E}_{s}\right\} \quad \sum_{s \in \mathcal{S}} U_{\beta}\left(r_{s}\right) \\
\text { s.t. } & \sum_{l \in \mathcal{O}(s)} R_{l}-\sum_{l \in \mathcal{I}(s)} R_{l}=r_{s}, \quad s \in \mathcal{S} \\
& r_{s} \geq r_{s}^{\mathrm{LB}}, \quad s \in \mathcal{S} \\
& \sum_{l \in \mathcal{O}(s)} \epsilon_{l} R_{l}+\sum_{l \in \mathcal{I}(s)} \varepsilon_{l} R_{l} \leq \tilde{E}_{s}, \quad s \in \mathcal{S} \\
& \sum_{s \in \mathcal{S}} \tilde{E}_{s} \leq \tilde{E}_{\mathrm{tot}}=\frac{E_{\mathrm{tot}}}{T_{f}} \\
& \sum_{l \in \mathcal{M}_{k}} \frac{R_{l}}{C_{l}} \leq 1, \quad k=1, \ldots, K .
\end{array}
$$

The optimization problem (7a)-(7b) with $T=T_{f}$ and total energy $E_{\text {tot }}$ is equivalent to the case with fixed network lifetime $T=1$ and new total energy $\tilde{E}_{\text {tot }}=\frac{E_{\text {tot }}}{T_{f}}$. If $T_{f}$ is increased to $T_{f}^{\prime}, \frac{E_{\text {tot }}}{T_{f}^{\prime}}$ becomes smaller than $\frac{E_{\text {tot }}}{T_{f}}$. Therefore, the feasible set of $\left\{\left\{r_{s}\right\},\left\{R_{l}\right\},\left\{\tilde{E}_{s}\right\}\right\}$ 's that satisfies (8b)-(8f) with $T_{f}^{\prime}$ will be a subset of the feasible set that satisfies (8b)(8f) with $T_{f}$. So the optimal value of (8a) with $T_{f}^{\prime}$ will be no more than the optimal value of (8a) with $T_{f}$. This completes the proof.

Consider an example network in Fig. 1, which has 14 nodes and 42 unidirectional links ( 21 bidirectional links). The Sink Node is the traffic destination for all other nodes' traffic. The energy needed to transmit 1 unit of data on any link $l$ is set to be 1 unit of energy, while the energy needed to receive 1 unit of data on any link is set to be 0.2 unit of energy, i.e., $\epsilon_{l}=1, \varepsilon_{l}=0.2, \forall l$. The capacity of each link, i.e., $C_{l}$, is 20 units of rate. We plot the total network throughput (viewed as the network utility in (8a)) versus the (fixed) network lifetime $T$ for different values of minimum source rate requirement $r_{s}^{\mathrm{LB}}$ and total energy $E_{\text {tot }}$, and show the result in Fig. 2. It can be seen that for each value of $r_{s}^{\mathrm{LB}}$ and $E_{\mathrm{tot}}$, the network

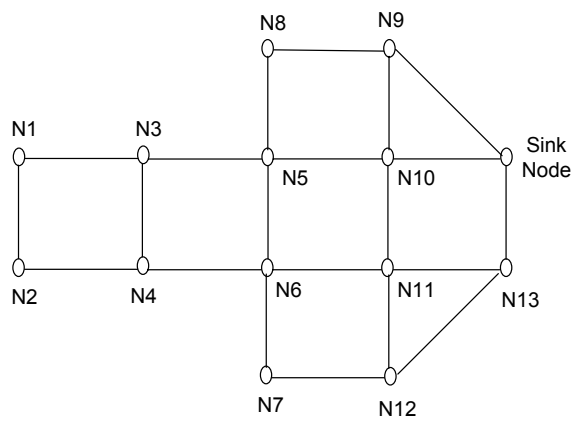

Fig. 1. An example of the network model.

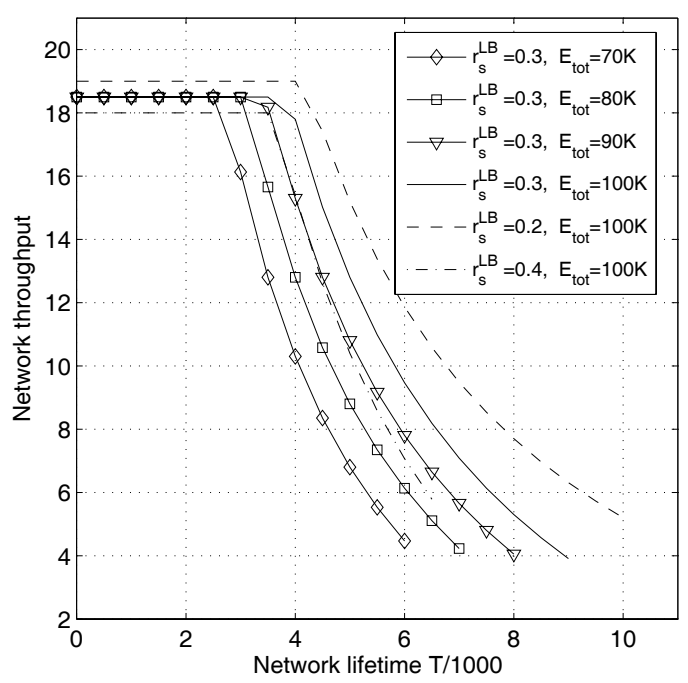

Fig. 2. Throughput versus fixed network lifetime $T$.

throughput first keeps constant, then starts to decrease after a threshold $T_{\text {threshold. }}$. The reason is as follows. If the network lifetime is small, each node has sufficient energy to transmit. Thus, the dominant factor to determine the network throughput is the contention constraints (which do not change). So the network throughput keeps constant for small values of network lifetime, as long as the energy supply is sufficient. After a threshold $T_{\text {threshold }}$, the energy supply of the network is not sufficient anymore, and thus turns to be the dominant factor to determine the network throughput. So the network throughput starts to decrease with an increase of network lifetime, since the energy supply becomes more stringent.

Using Theorem 1, the following algorithm for solving problem $(5 \mathrm{a})-(5 \mathrm{~g})$ is proposed.

- Step 1: Given $E_{\mathrm{tot}}$, determine the threshold point $T_{\text {threshold} \text {. }}$

- Step 2: Set $T=\max \left\{T_{\text {threshold }}, T_{\min }\right\}$.

- Step 3: Solve (5a)-(5g) with $T$ determined as in Step 2. The bisection search method can be used to determine $T_{\text {threshold }}$ in Step 1 without the need for an exhaustive search. However, the bisection search only provides an approximate value of $T_{\text {threshold }}$ within certain accuracy to the actual value. Particularly, two variables $T_{1}$ and $T_{2}$ are used in the bisection search, where $T_{1}$ is first fixed at a very small value, and the 
corresponding network throughput ${ }^{5}$ is denoted as $U_{1}$, and $T_{2}$ is taken to be large enough such that the corresponding network throughput $U_{2}<U_{1}$. Set $T_{1}:=\frac{T_{1}+T_{2}}{2}$ if network throughput associated with network lifetime $\frac{T_{1}+T_{2}}{2}$ is equal to $U_{1}$, or set $T_{2}:=\frac{T_{1}+T_{2}}{2}$ otherwise. This procedure is repeated until the difference between $T_{1}$ and $T_{2}$ is small enough (say, less than a pre-selected threshold value that defines the desired accuracy). Finally, we set $T_{\text {threshold }}:=\frac{T_{1}+T_{2}}{2}$, which is the approximated point when the throughput starts to decrease as shown in Fig. 2. Therefore, depending on the accuracy of the proposed design, a smaller or larger number of sub-problems need to be solved in Step 1. Clearly, with some initial ideas on the value $T_{\text {threshold }}$, the complexity of the aforementioned process can be further reduced.

For the optimization problem in Step 3, the following Theorem proves its convexity.

THEOREM 2: The optimization problem $(5 \mathrm{a})-(5 \mathrm{~g})$ with fixed $T$ is convex.

Proof: The utility function $U_{\beta}\left(r_{s}\right)$ given in (6) is concave on source rate variables $r_{s}, \forall s \in \mathcal{S}$ [10]. It is easy to show that the constraints in $(5 \mathrm{~b})-(5 \mathrm{~g})$ with fixed $T$ are linear constraints with respect to the variables $r_{s}, R_{l}, E_{s}, \forall l, s$. Thus, the optimization problem is convex.

Theorems 1 and 2 indicate that the optimization problem (5a) $-(5 \mathrm{~g})$ is approximately equivalent to a two-step convex problem. Generally this two-step convex problem should be solved in a centralized manner. Therefore, for a large-size network with a large number of variables (such as a sensor network), the computational complexity to solve the problem may be high. However, this drawback can be compensated by the following two facts: 1) We have proved that the subproblems in the proposed design are convex, and thus can be solved with limited complexity, since software packages are available to solve large-scale convex problems. 2) Although a centralized algorithm has high computational complexity, and thus, is not scalable for a large-size sensor network, we can partition the network into clusters (as discussed in [11] and references therein), and apply the centralized algorithm in each cluster. Overall, the proposed algorithm is targeted for a small- or medium-size network, or a cluster in a large-size network.

\section{NUMERICAL RESUltS}

Consider a multi-hop network as shown in Fig. 1. Three different scenarios are tested: network throughput maximization with all nodes having equal energy, network throughput maximization with energy distribution, and max-min fairness optimization with energy distribution, referred to as Schemes 1,2 , and 3 , respectively.

First set $\epsilon_{l}=1$ unit of energy, $\varepsilon_{l}=0.2$ unit of energy, and $C_{l}=20$ units of rate, $\forall l$. The minimum network lifetime requirement is $T_{\min }=5000$ units of time. The minimum source rate requirement for each node is $r_{s}^{\mathrm{LB}}=0.1$ or 0.2 unit of rate. The network throughput is obtained for the three schemes with different $E_{\text {tot }}$ values, shown in Fig. 3. It can be

\footnotetext{
${ }^{5}$ Note that any network throughput associated with a fixed network lifetime $T$ in the bisection search is obtained by solving the problem (8a)-(8f) with the fixed $T$.
}

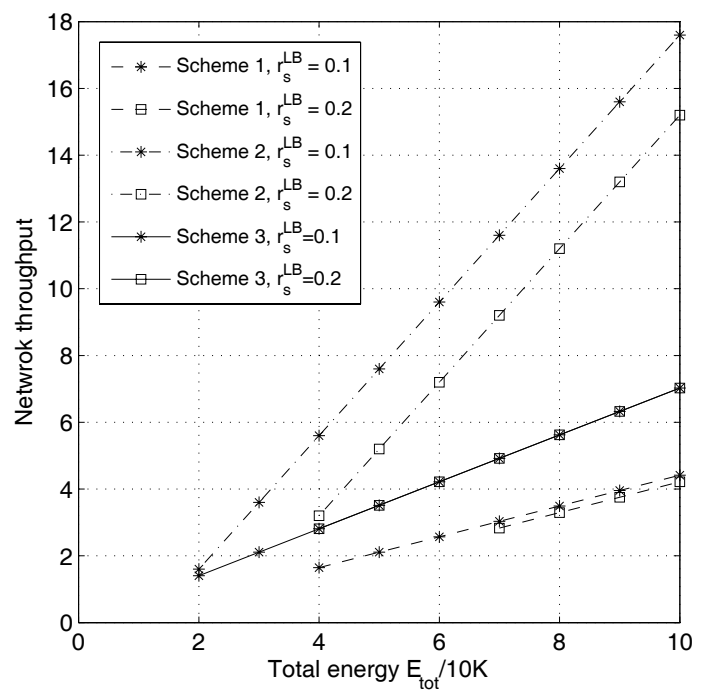

Fig. 3. Throughput versus total available energy $E_{\text {tot }}$.

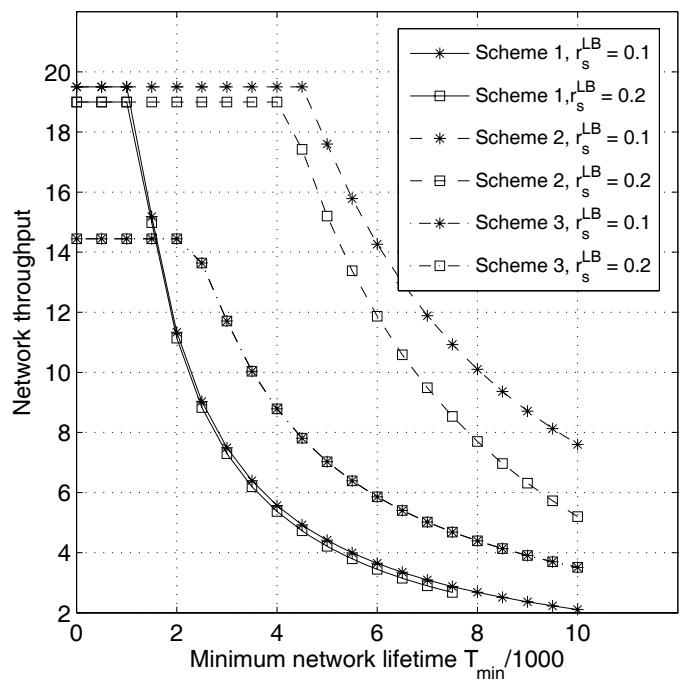

Fig. 4. Network throughput versus network lifetime requirement $T_{\min }$ when $E_{\text {tot }}=100 \mathrm{~K}$.

seen that Scheme 2 leads to the maximum network throughout, while Scheme 1 leads to the least. Next, we fix $T_{\min }=5000$ units of time, $r_{s}^{\mathrm{LB}}=0.2$ unit of rate, and $E_{\text {tot }}=100 \mathrm{~K}$ units of energy. Table I shows the assigned energy (i.e., $E_{s}$ ) and source rate (i.e., $r_{s}$ ) of each node. In Scheme 1, all the nodes have the same energy assignment. Scheme 2 achieves the largest network throughput, and at the same time, the largest variance in the source rates of the nodes. Scheme 3 leads to the best fairness performance since the source rates of all the nodes are the same. The variance in the assigned energy of the nodes in Scheme 3 is because of the variance of the relay traffic load at the nodes. In other words, a node may need more energy if it needs to relay more traffic.

The effect of users' QoS demands on the network throughput is also investigated. Fig. 4 displays the network throughput 
TABLE I

Assigned ENERGy AND SOURCE RATE AT EACH NODE WHEN $T_{\min }=5000, r_{s}^{\mathrm{LB}}=0.2 \mathrm{AND} E_{\mathrm{tot}}=100 \mathrm{~K}$.

\begin{tabular}{|c|c|c|c|c|c|c|c|c|c|c|c|c|c|c|}
\hline \multicolumn{2}{|c|}{ Node ID } & N1 & N2 & N3 & $\mathrm{N} 4$ & $\mathrm{~N} 5$ & N6 & N7 & N8 & N9 & N10 & N11 & N12 & N13 \\
\hline \multirow{3}{*}{ Energy } & Scheme 1 & 7.7 & 7.7 & 7.7 & 7.7 & 7.7 & 7.7 & 7.7 & 7.7 & 7.7 & 7.7 & 7.7 & 7.7 & 7.7 \\
\hline & Scheme 2 & 1.4 & 1.0 & 3.2 & 1.8 & 5.3 & 2.4 & 1.0 & 1.0 & 23.9 & 28.2 & 2.8 & 2.2 & 25.9 \\
\hline & Scheme 3 & 3.6 & 2.7 & 8.1 & 5.0 & 13.3 & 7.1 & 2.7 & 2.7 & 6.0 & 23.2 & 8.3 & 6.0 & 11.4 \\
\hline \multirow{3}{*}{ Rate } & Scheme 1 & 0.2 & 0.2 & 0.2 & $\overline{0.2}$ & 0.2 & 0.2 & 0.2 & 0.2 & 0.9 & 0.7 & 0.2 & 0.2 & 0.7 \\
\hline & Scheme 2 & 0.2 & 0.2 & 0.2 & 0.2 & 0.2 & 0.2 & 0.2 & 0.2 & 4.5 & 4.3 & 0.2 & 0.2 & 4.4 \\
\hline & Scheme 3 & 0.5 & 0.5 & 0.5 & 0.5 & 0.5 & 0.5 & 0.5 & 0.5 & 0.5 & 0.5 & 0.5 & 0.5 & 0.5 \\
\hline
\end{tabular}

when $E_{\mathrm{tot}}=100 \mathrm{~K}$ units of energy, and each node's source rate demand $r_{s}^{\mathrm{LB}}$ is 0.1 and 0.2 unit of rate, respectively. It can be seen that the optimized total network throughput keeps nonincreasing with the increase of the users' source rate demand. This can be explained as follows. In the optimization problem (5a)-(5g), the constraint (5c) becomes more stringent with an increase of the source rate demand. Thus, the feasible set of $\left\{\left\{r_{s}\right\},\left\{R_{l}\right\},\left\{E_{s}\right\}, T\right\}$ 's will be a subset of the feasible set in the case if the source rate demand does not increase. Thus, an increase of source rate demand results in the same (e.g., Scheme 3 in the example) or smaller (e.g., Schemes 1 and 2 in the example) optimal value of the network utility.

\section{Further Discussions}

In the NUM formulation in Section III, our target is to maximize the network utility, within the minimum required network lifetime. An alternative and interesting formulation is to maximize the network utility within the achievable network lifetime, while guaranteeing that the achievable network lifetime is at least the minimum required value. For this alternative case, the energy supply at node $s$ is fixed as $E_{s}$. Then we obtain the following NUM problem:

$$
\begin{array}{ll}
\max _{\left\{r_{s}\right\},\left\{R_{l}\right\}, T} T \sum_{s \in \mathcal{S}} U_{\beta}\left(r_{s}\right) \\
\text { s.t. } & \sum_{l \in \mathcal{O}(s)} R_{l}-\sum_{l \in \mathcal{I}(s)} R_{l}=r_{s}, \quad s \in \mathcal{S} \\
& r_{s} \geq r_{s}^{\mathrm{LB}}, \quad s \in \mathcal{S} \\
& {\left[\sum_{l \in \mathcal{O}(s)} \epsilon_{l} R_{l}+\sum_{l \in \mathcal{I}(s)} \varepsilon_{l} R_{l}\right] T \leq E_{s}, s \in \mathcal{S}} \\
& T \geq T_{\min } \\
& \sum_{l \in \mathcal{M}_{k}} \frac{R_{l}}{C_{l}} \leq 1, \quad k=1, \ldots, K .
\end{array}
$$

TheOrem 3: The optimization problem (9a)-(9f) is quasiconvex.

Proof: A new variable is defined as $B=1 / T$. Then the problem (9a)-(9f) can be equivalently rewritten as

$$
\begin{array}{ll}
\min _{\left\{r_{s}\right\},},\left\{R_{l}\right\}, B & -\frac{1}{B} \sum_{s \in \mathcal{S}} U_{\beta}\left(r_{s}\right) \\
\text { s.t. } & \sum_{l \in \mathcal{O}(s)} \epsilon_{l} R_{l}+\sum_{l \in \mathcal{I}(s)} \varepsilon_{l} R_{l} \leq B E_{s}, s \in \mathcal{S}(10 \mathrm{~b}) \\
& B \leq 1 / T_{\min } \\
& \text { Constraints (9b), (9c), (9f). }
\end{array}
$$

The constraints (10b)-(10d) in the above problem are linear on variables $\left\{r_{s}\right\},\left\{R_{l}\right\}$ and $B$. In order to prove that the objective function (10a) is quasiconvex, we consider the following $\alpha$ sublevel set $(\alpha \in \mathbb{R})$

$$
\begin{aligned}
\mathcal{N} & =\left\{\left\{r_{s}\right\},\left\{R_{l}\right\}, B \mid-\frac{1}{B} \sum_{s \in \mathcal{S}} U_{\beta}\left(r_{s}\right) \leq \alpha\right\} \\
& =\left\{\left\{r_{s}\right\},\left\{R_{l}\right\}, B \mid-\sum_{s \in \mathcal{S}} U_{\beta}\left(r_{s}\right)-\alpha B \leq 0\right\} .
\end{aligned}
$$

Since $\sum_{s \in \mathcal{S}} U_{\beta}\left(r_{s}\right)$ is concave, $-\sum_{s \in \mathcal{S}} U_{\beta}\left(r_{s}\right)$ is convex. And $\alpha B$ is linear on $B$. So the set $\mathcal{N}$ is convex. And thus, the objective function (10a) is quasiconvex [12]. This completes the proof.

\section{CONCLUSION}

Cross-layer approaches are powerful tools for the design of multi-hop wireless networks supporting multimedia services with QoS guarantee. In this letter, the joint design of MAC, routing, and energy distribution in a multi-hop network is formulated as a nonconvex and nonlinear NUM optimization problem, and a near optimal solution is developed. It is also shown that the problem of maximizing sum utility within achievable network lifetime is quasiconvex. This research can help facilitate node configuration and link/network layer protocol design in multi-hop wireless networks, such as wireless mesh networks, mobile ad hoc networks, and wireless sensor networks.

\section{ACKNOWLEDGEMENTS}

The authors would like to thank the anonymous reviewers for their constructive comments and suggestions which helped to improve the quality of the manuscript.

\section{REFERENCES}

[1] H. T. Cheng, H. Jiang, and W. Zhuang, "Distributed medium access control for wireless mesh networks," Wireless Commun. and Mobile Comput., vol. 6, no. 6, pp. 845-864, Sept. 2006.

[2] X. Shen, W. Zhuang, H. Jiang, and J. Cai, "Medium access control in ultra-wideband wireless networks," IEEE Trans. Veh. Technol., vol. 54, no. 5, pp. 1663-1677, Sept. 2005.

[3] A. Abdrabou and W. Zhuang, "A position-based QoS routing scheme for UWB mobile ad hoc networks," IEEE J. Select. Areas Commun., vol. 24, no. 4, pp. 850-856, Apr. 2006.

[4] S.-J. Kim, X. Wang, and M. Madihian, "Distributed joint routing and medium access control for lifetime maximization of wireless sensor networks," IEEE Trans. Wireless Commun., vol. 6, no. 7, pp. 2669-2677, July 2007.

[5] M. L. Sichitiu and R. Dutta, "On the lifetime of large wireless sensor networks with multiple battery levels," Ad Hoc \& Sensor Wireless Networks, vol. 4, no. 1-2, pp. 69-96, 2007.

[6] T. Nandagopal, T.-E. Kim, X. Gao, and V. Bharghavan, "Achieving MAC layer fairness in wireless packet networks," in Proc. ACM MOBICOM'00, pp. 87-98. 
[7] Z. Fang and B. Bensaou, "Fair bandwidth sharing algorithms based on game theory frameworks for wireless ad-hoc networks," in Proc. IEEE INFOCOM'04, pp. 1284-1295.

[8] L. Chen, S. H. Low, and J. C. Doyle, "Joint congestion control and media access control design for ad hoc wireless networks," in Proc. IEEE INFOCOM'05, pp. 2212-2222.

[9] F. P. Kelly, A. K. Maulloo, and D. K. H. Tan, "Rate control in communication networks: shadow prices, proportional fairness and stability," $J$. Operational Research Society, vol. 49, no. 3, pp. 237-252, Mar. 1998.
[10] J. Mo and J. Walrand, "Fair end-to-end window-based congestion control," IEEE/ACM Trans. Networking, vol. 8, no. 5, pp. 556-567, Oct. 2000.

[11] O. Younis, M. Krunz, and S. Ramasubramanian, "Node clustering in wireless sensor networks: Recent developments and deployment challenges," IEEE Network, vol. 20, no. 3, pp. 20-25, May/June 2006.

[12] S. Boyd and L. Vandenberghe. Convex Optimization. Cambridge University Press, 2004. 